\title{
The 2016 WHO Classification of Tumours of the Central Nervous System: The Major Points of Revision
}

\author{
Takashi KOMORI ${ }^{1}$ \\ ${ }^{1}$ Department of Laboratory Medicine and Pathology (Neuropathology), \\ Tokyo Metropolitan Neurological Hospital, Tokyo, Japan
}

\begin{abstract}
The updated 2016 edition of the World Health Organization (WHO) Classification of Tumours of the Central Nervous System (CNS) uses molecular parameters and the histology to define the main tumor categories for the first time. This represents a shift from the traditional principle of using neuropathological diagnoses, which are primarily based on the microscopic features, to using molecularly-oriented diagnoses. Major restructuring was made with regard to diffuse gliomas, medulloblastomas and other embryonal tumors. New entities that are defined by both the histological and molecular features include glioblastoma, isocitrate dehydrogenase (IDH)-wildtype and glioblastoma, IDH-mutant; diffuse midline glioma, H3 K27M-mutant; RELA fusion-positive ependymoma; medulloblastoma, wingless (WNT)-activated and medulloblastoma, sonic hedgehog (SHH)-activated; and embryonal tumor with multilayered rosettes, C19MC-altered. In addition, some entities that are no longer diagnostically relevant-such as CNS-primitive neuroectodermal tumor-have been deleted from this updated edition. The WHO2016 certainly facilitates clinical and basic research to improve the diagnosis of brain tumors and patient care.
\end{abstract}

Key words: World Health Organization (WHO), classification, histology, genetics, new entities

\section{Introduction}

In the past decades, the traditional approach to the diagnosis of tumors of the central nervous system, which was primarily based on the microscopic features, has shifted to a molecularly-oriented approach. This change has been driven by genetic as well as epigenetic discoveries. ${ }^{1)}$ The updated 4th edition of the World Health Organization (WHO) Classification of Tumours of the Central Nervous System (WHO2016) has opened the door to a molecular era that the neuropathology/ neuro-oncology community has never faced. ${ }^{2-4)}$

Since Baily \& Cushing introduced the histogenetic classification of the tumors of the central nervous system in 1926, ${ }^{5)}$ the basic concept of classification has remained essentially unchanged, regardless of developments in the methods that are applied to the analysis of human tissue. Tumors are classified according to their similarity to the constituent cells of the central nervous system, such as astrocytes, oligodendrocytes and ependymal cells and are further sub-classified according to the presumed level of differentiation, which is determined based on morphological irregularities in comparison to their normal counterpart.

Received January 17, 2017; Accepted February 23, 2017
Such similarities have been depicted by microscopic features on hematoxylin and eosin-stained sections, immunohistochemistry corresponding to lineagespecific proteins such as glial fibrillary acidic protein for the astrocytic lineage and ultrastructural findings that characterize histogenetic differentiation. Mitosis and cell cycle-specific antigens are used as markers to evaluate the proliferation activity and biological behavior (the WHO grading system). ${ }^{6)}$

These histogenetic classification and grading systems have been valid for near a century because they were roughly correlated with the prognosis and have remained beneficial to determining treatment strategies, including adjuvant therapies. Nonetheless, for the past 2 decades, these classification and grading systems have been challenged by genetic/epigenetic discoveries in at least three areas. First, histogenetic classification is no longer valid since it is clear that various differentiations can co-exist within the tissue of a single tumor. For example, astrocytic, ${ }^{7)}$ oliogdendroglial ${ }^{8)}$ and ependymal tumors ${ }^{9-13)}$ can co-exist with mature neurons and ependymal differentiation can be found across many different lineages beyond ependymomas. Second, the prognoses are less correlated with the WHO grade than the major molecular profiles. ${ }^{14-21)}$ Third, when making a pathological diagnosis, inter-observer 
differences are no longer acceptable since molecular testing offers better objectivity and reproducibility than subjective microscopic observation. ${ }^{22-24)}$

One of the first genetic alterations that led to the transformation of the diagnostic approach was a codeletion of chromosome $1 \mathrm{p}$ and $19 \mathrm{q}$ in oligodendroglioma. ${ }^{14,25,26)}$ The term of oligodendroglioma was coined in remembrance of normal oligodendroglia, as defined by Baily \& Cushing in the 1920s. ${ }^{5)}$ Nonetheless, true oligodendroglial differentiation, such as myelin formation, has never been identified in ultrastructural studies and neither myelin-related protein nor messenger RNA has been consistently demonstrated in oligodendroglioma. Instead, oligodendroglia-like cells are often found in various neuroepithelial tumors with diverse differentiation and biological behavior-a situation that has caused significant diagnostic difficulties. ${ }^{27-31)}$ On the other hand, the $1 \mathrm{p} / 19 \mathrm{q}$ codeletion is well-correlated with both classic oligodendroglioma morphology and its clinical, radiological and biological characteristics, ${ }^{17,18,32,33)}$ all of which indicate that gliomas harboring $1 \mathrm{p} / 19 \mathrm{q}$ fall into a single entity.

Isocitrate dehydrogenase 1 and 2 (IDH1 and IDH2, respectively) mutations are another type of genetic alteration that has had an impact on tumor classification. ${ }^{16-18,21,34)}$ These mutations are found exclusively in infiltrating astrocytomas and oligodendrogliomas but not in circumscribed astrocytomas or ependymomas. ${ }^{34-36)}$ A number of studies have shown that these mutations are strong prognostic makers and that they may well be the most upstream genetic event in the tumorigenesis of infiltrating astrocytomas and oligodendrogliomas. ${ }^{37)}$ The discovery of IDH1/2 mutations is significant because it provides further evidence to rebut the traditional histogenetic classification systems and because it provides a common frame for two different entities beyond presumed lineages.

The incorporation of the sonic hedgehog (SHH) and wingless (WNT) pathways in medulloblastomas also has prognostic and predictive implications. ${ }^{38,39)}$ Medulloblastoma with alterations in the WNT pathways is associated with a significantly indolent prognosis while medulloblastoma with group 3 and 4 has the worst prognosis. Most WNT-activated tumors exhibit classic medulloblastoma morphology but not all tumors with classic medulloblastoma morphology show WNT activation. Thus, medulloblastomas are classified according to their genetic and histological features.

\section{The basic principles of the revision of WHO2016 The Haarlem consensus guidelines}

Before the consensus meeting for WHO2016 in Heidelberg, a meeting was held in Haarlem, the
Netherlands, to discuss how non-histological data such as molecular information could be incorporated into the next WHO classification of brain tumors. A consensus was reached that molecular information should be incorporated into the next WHO classification in accordance with a set of guidelines provided by the "International Society of NeuropathologyHaarlem meeting". ${ }^{40)}$ The main recommendations were that (i) diagnostic entities should be defined as narrowly as possible in order to optimize interobserver reproducibility, the clinicopathological predictions and therapeutic planning; (ii) diagnoses should be "layered" with a histological classification, the WHO grade and molecular information should be listed below an "integrated diagnosis" (Table 1); and (iii) determinations should be made for each tumor entity as to whether molecular information is required, suggested, or not needed for its definition.

\section{Histology-based molecular classification}

In WHO2016, the conventional histological results obtained using H\&E-stained sections remain the initial stratifier. After determining the major category (such as infiltrating glioma, neuronal tumor or embryonal tumor) based on the histology, a subset is applied based on the results of molecular testing (Table 2). ${ }^{40,411}$

Table 1 Reporting format

\begin{tabular}{lll}
\hline & Nomenclature & Example \\
\hline Layer 1 & $\begin{array}{l}\text { Integrated diagnosis } \\
\text { (incorporating all tissue- } \\
\text { based information) }\end{array}$ & $\begin{array}{l}\text { Astrocytoma, } \\
\text { IDHmt }\end{array}$ \\
Layer 2 & Histological classification & Oligoatrocytoma \\
Layer 3 & $\begin{array}{l}\text { WHO grade (reflecting } \\
\text { natural history) }\end{array}$ & II \\
Layer 4 & Molecular information & $\begin{array}{l}\text { IDH1R132H+, } \\
\text { 1p/19q non-deleted, } \\
\end{array}$ \\
& & p53+, ATRX loss \\
\hline
\end{tabular}

IDH: isocitrate dehydrogenase, mt: mutant.

Table 2 Tumor categories requiring molecular information for classification

\begin{tabular}{lll}
\hline & $\begin{array}{l}\text { Adults or } \\
\text { supratentorial } \\
\text { location }\end{array}$ & $\begin{array}{l}\text { Child and } \\
\text { adolescence or } \\
\text { infratentorial } \\
\text { location }\end{array}$ \\
\hline $\begin{array}{l}\text { Diffuse astrocytic } \\
\text { and oligodendroglial } \\
\text { tumors }\end{array}$ & $\begin{array}{l}\text { IDH1/2 1p19q } \\
\text { codeletion }\end{array}$ & H3 K27M \\
$\begin{array}{l}\text { Ependymal tumors } \\
\text { Embryonal tumors }\end{array}$ & RELA fusion & \\
\hline $\begin{array}{l}\text { IDH: isocitrate dehydrogenase. } \\
\text { WNI-1, C19MC }\end{array}$
\end{tabular}

Neurol Med Chir (Tokyo) 57, July, 2017 
In terms of discordant results such as "a diffuse glioma that histologically appears astrocytic but proves to have IDH mutation and $1 \mathrm{p} / 19 \mathrm{q}$ codeletion" or "a tumor that resembles oligodendroglioma by light microscopy but has IDH, ATRX and TP53 mutations in the setting of intact $1 \mathrm{p}$ and $19 \mathrm{q}$ ", it is clearly stated in the review article written by the senior editors of the WHO2016 that the genotype trumps the histological phenotype. ${ }^{3)}$ Nevertheless, it remains possible that 'not otherwise specified (NOS)' designations can be applied to discordant examples since the WHO2016 is predicated on the basis of combined phenotypic and genotypic classification and on the generation of "integrated" diagnoses. ${ }^{40)}$

\section{The 'not otherwise specified' (NOS) status}

In accordance with the Haarlem guidelines, the NOS status was introduced in WHO2016 to define entities as narrowly as possible. NOS is applied when (i) genetic testing is not available, (ii) genetic testing does not show diagnostic genetic alterations that are compatible with the histological findings or (iii) when there is uncertainty about a tumor's architectural or cytological features due to insufficient tissue sampling or the presence of tissue artifacts. ${ }^{2,3)}$

\section{The Major Points of Revision}

\section{The revised entities and variants are listed in Table 3.}

Oligodendrogliomas: The histology of oligodendroglioma has to be 'classic', since this nomenclature is intended to define 1p19q codeleted glioma. More than $90 \%$ of classic oligodendrogliomas show IDH mutation and 1p19q codeletion; which is now considered a genetic signature of oligodendroglioma. ${ }^{3)}$ Given the high frequency of $\mathrm{R} 132 \mathrm{H}$ mutations in $I D H 1$ that are detectable by immunohistochemistry, ${ }^{42,43)}$ molecular testing for another locus in IDH1/2 may be required in less than $10 \%$ of classic oligodendrogliomas. ${ }^{44)}$ If it becomes anaplastic, the classic histology will be unclear and genetic testing for codeletion will be mandatory in that setting. When a classic oligodendroglioma is classified as IDH wildtype, the final diagnosis is oligodendroglioma, NOS, after other mimicking entities are excluded (Table 4). ${ }^{3)}$

\section{Diffuse astrocytomas (Fig. 1)}

After the histological confirmation of astrocytoma, the second stratifier for adult patients is the presence or absence of IDH1 or IDH2 mutations. If TP53 as well as ATRX mutations (both of which are mutually exclusive to $1 \mathrm{p} 19 \mathrm{q}$ codeletion) are present in IDHmutant gliomas, the diagnosis of oligodendroglioma is immediately excluded. ${ }^{41,45-47)}$ Either TP53 or ATRX mutations can be detected by immunohistochemistry (Table 5). If the tumor is located in the thalamus or pons, an H3 K27M mutation, ${ }^{48-50)}$ which is mutually exclusive of $I D H 1 / 2$ mutations, should be considered. When a $1 \mathrm{p} 19 \mathrm{q}$ codeletion is present, the tumor is further classified as oligodendroglioma, regardless of the histology. All IDH1/2-mutant gliomas without codeletions are now classified as astrocytoma. Oligoastrocytoma, anaplastic oligoastrocytoma and glioblastoma with an oligodendroglial component were deleted from the classification, since they are no longer genetically relevant. ${ }^{31)}$ Gliomas in pediatric patients, particularly patients under ten years of age, are unlikely to possess $I D H 1 / 2$ mutations or $1 \mathrm{p} 19 \mathrm{q}$ codeletions ${ }^{51-53)}$ and generally fall into the category of diffuse or anaplastic astrocytoma, IDH wildtype. The nosological positions of pediatric- and adulttype IDH-wildtype gliomas are currently ambiguous; most of the latter behave like glioblastoma, ${ }^{54)}$ and are transcribed in italics. Although some data suggest that the prognosis of WHO grade II IDH-mutant glioma does not differ from that of WHO grade III IDH-mutant glioma, ${ }^{20)}$ the grading scheme was not changed in this revision. Nonetheless some amendments will be required in the next revision.

\section{Glioblastomas}

The definition of this nomenclature remains histological rather than genetic, i.e. a high-grade glioma with predominantly astrocytic differentiation, featuring nuclear atypia, cellular pleomorphism as well as microvascular proliferation and/ or necrosis. ${ }^{2)}$ Depending on the absence or presence of IDH1/2 mutations, glioblastomas are divided into glioblastoma, IDH-wildtype, which corresponds to clinically-defined primary or de novo glioblastoma, and glioblastoma, IDH-mutant, which corresponds to so-called secondary glioblastoma. ${ }^{55)}$ It was decided that the terms, primary and secondary, would not be used in WHO2016, since they are clinically defined. Glioblastomas with negative R132H IDH1 immunohistochemistry are quite important clinically and are considered to be equivalent to glioblastoma, IDH-wildtype in patients older than 55 years of age, since no mutations other than IDH1 R132H have been reported in glioblastomas in that age group. ${ }^{3)}$

One new glioblastoma variant is epitheliod glioblastoma, which has been designated as rhabdoid or epithelioid/rhabdoid. ${ }^{56,57)}$ To avoid confusion with true rhabdoid tumors such as atypical teratoid/ rhabdoid tumor (AT/RT), which harbors INI1 or BRG1 mutations, the term 'rhabdoid' is abandoned to describe this variant; in approximately half of the cases, it lacks either mutation but harbors a $B R A F$ V600E mutation. ${ }^{57)}$ 
Table 3 Major points of revision

Diffuse astrocytic and oligodendroglial tumours

Diffuse astrocytoma, IDH mutant

Gemistocytic astrocytoma, IDH mutant

Diffuse astrocytoma IDH wildtype

Diffuse astrocytoma, NOS

Anaplastic astrocytoma, IDH mutant

Anaplastic astrocytoma, IDH wildtype

Anaplastic astrocytoma, NOS

Glioblastoma, IDH wildtype

Giant cell glioblastoma

Gliosarcoma

Epithelioid glioblastoma

Glioblastoma, IDH mutant

Glioblastoma, NOS

Diffuse midline glioma, H3-K27M mutant

Oligodendroglioma, IDH mutant and 1p/19q codeleted

Oligodendroglioma, NOS

Anaplastic oligodendroglioma, IDH mutant and 1p/19q codeleted

Anaplastic oligodendroglioma, NOS

Oligoastrocytoma, NOS

Anaplastic oligoastrocytoma, NOS

Other astrocytic tumours

Pilocytic astrocytoma

Pilomyxoid astrocytoma

Subependymal giant cell astrocytoma

Pleomorphic xanthoastrocytoma

Anaplastic pleomorphic xanthoastrocytoma
Embryonal tumours

Medulloblastoma, genetically defined

Medulloblastoma, WNT activated

Medulloblastoma, SHH activated, TP53 mutated

Medulloblastoma, SHH activated, TP53 wildtype

Medulloblastoma, non-WNT/non-SHH

Medulloblastoma, group 3

Medulloblastoma, group 4

Medulloblastoma, histologically defined

Medulloblastoma, classic

Medulloblastoma, desmoplastic/nodular

Medulloblastoma with extensive nodularity

Medulloblastoma, large cell/anaplastic

Medulloblastoma, NOS

Embryonal tumour with multilayered rosettes, C19MC altered

Embryonal tumour with multilayered rosettes, NOS

Medulloepithelioma

CNS neuroblastoma

CNS ganglioneuroblastoma

CNS embryonal tumour, NOS

Atypical teratoid/rhabdoid tumour

CNS embryonal tumour with rhabdoid features

Table 4 WHO grade II adult diffuse gliomas

\begin{tabular}{|c|c|c|c|}
\hline & Astrocytoma histology & Oligodendroglioma histology & $\begin{array}{l}\text { Oligoastrocytoma or } \\
\text { ambiguous histology }\end{array}$ \\
\hline $\begin{array}{l}\text { IDHmt, 1p19q-nondel, ATRX } \\
\text { loss }\end{array}$ & Diffuse astrocytoma, IDHmt & Diffuse astrocytoma, IDHmt & Diffuse astrocytoma, IDHmt \\
\hline $\begin{array}{l}\text { IDHmt, 1p19q-codel, ATRX } \\
\text { intact }\end{array}$ & $\begin{array}{l}\text { Oligodendroglioma, IDHmt } \\
\& 1 p 19 q \text { codel }\end{array}$ & $\begin{array}{l}\text { Oligodendroglioma, IDHmt \& } \\
\text { 1p19q codel }\end{array}$ & $\begin{array}{l}\text { Oligodendroglioma, IDHmt } \\
\& 1 p 19 q \text { codel }\end{array}$ \\
\hline IDHwt & Diffuse astrocytoma, IDHwt & Oligodendroglioma, NOS & Diffuse astrocytoma, IDHwt \\
\hline
\end{tabular}

IDH: isocitrate dehydrogenase. 

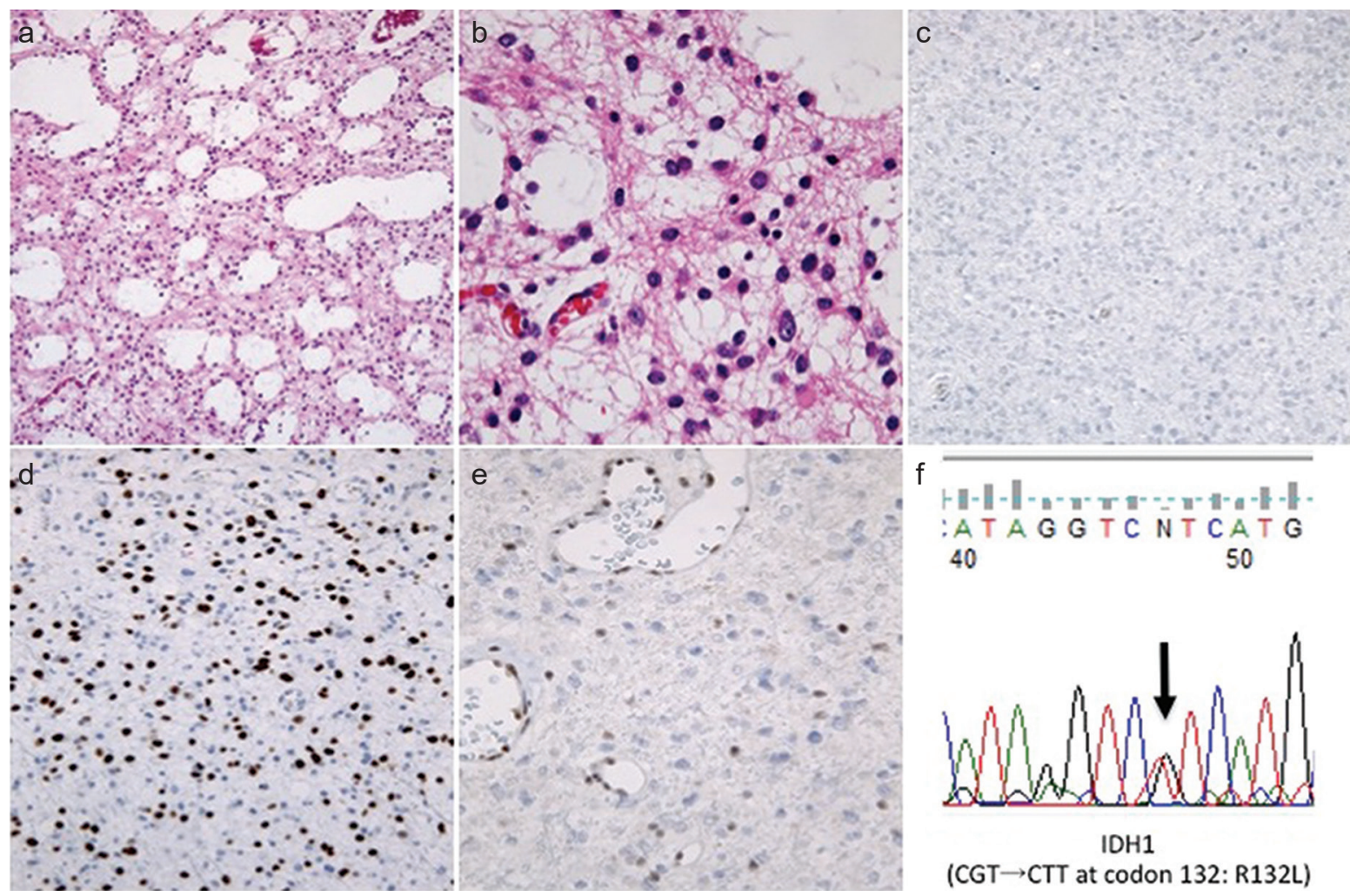

Fig. 1 Anaplastic astrocytoma, WHO2016. (a) Relatively uniform oval to elongated nuclei are evident in microcystic background. (b) In a higher-powered view, some nuclei are naked without apparent cytoplasmic processes while some pose fibrillary processes, nuclei showing irregularity and hyperchromasia. Such features correspond to anaplastic oligoastrocytoma in the previous WHO classification. (c) IDH1R132H immunohistochemistry is negative. (d) p53 is diffusely positive, suggesting TP53 mutation. (e) ATRX immunoreactivity is lost in tumor cells but intact in endothelial cells. (f) Positive p53 and negative ATRX suggest the presence of IDH mutation. Sanger sequence reveals a R132L mutation in IDH1.

Table 5 Immunohistochemical surrogates for molecular alterations required in WHO2016

\begin{tabular}{llll}
\hline Antibody & Clone & Molecular alterations & Positive pattern \\
\hline IDH1R132H & H09 & Arg to His at 132 in IDH1 & Cytoplasmic staining \\
ATRX & HPA001906 & ATRX mutations & Loss of nuclear expression \\
p53 & DO-7 & TP53 mutations & More than 10\% of nuclear expression \\
BRAFV600E & VE1 & Val to Glu at 600 in BRAF & Cytoplasmic staining \\
H3 K27M & ABE419 & Lys to Met at 27 in H3.1 or H3.3 & Nuclear staining \\
L1CAM & OTI2C7 & $\begin{array}{l}\text { Correlation with C11orf95-RELA } \\
\text { fusion and NF-Kappa B activation }\end{array}$ & Diffuse cytoplasmic staining \\
$\beta$-catenin & Ab610154 & Medulloblastoma, WNT-activated & Diffuse nuclear staining \\
GAB1 & Ab133486 & Medulloblastoma, SHH-activated & Cytoplasmic staining \\
LIN28A & A177, \#3978 & ETMR & Diffuse cytoplasmic staining \\
\hline
\end{tabular}

IDH: isocitrate dehydrogenase.

\section{Pediatric diffuse astrocytomas and oligodendrogliomas}

These tumors, which share a common histology, are grouped with their adult counterparts in WHO2016, despite the clear difference in clinical behavior between the tumors in pediatric and adult patients.
This is partly because WHO2016 is an upgrade of the previous edition, which did not allow the coining of a new framework, such as pediatric glioma subgroup within the classification but also because no single genetic alteration is sufficient to create a new entity in these pediatric gliomas. ${ }^{51,52)}$ The only 

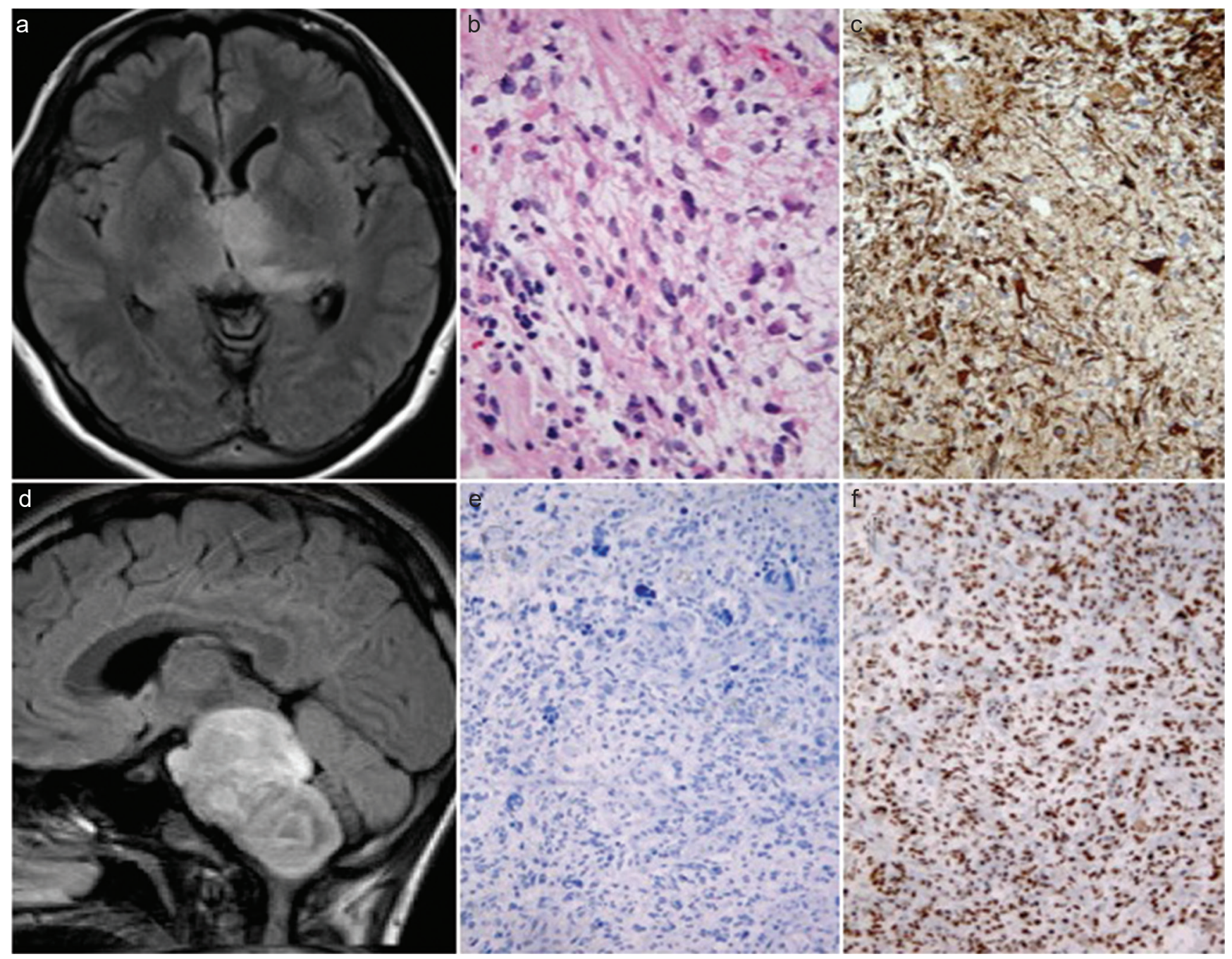

Fig. 2 Diffuse midline glioma, H3 K27M-mutant. (a) Axial FLAIR MRI shows an ill-defined high intensity area in the left thalamus. (b) Thalamic tumor shows diffuse astrocytic morphology with anaplasia. (c) The tumor cells show strong GFAP expression. (d) Sagittal FLAIR MRI shows a diffusely infiltrating pontine glioma expanding the pons. (e) IDH1 R132H immunohistochemistry is negative. (f) Strong nuclear staining for K27M-mutant H3 is present.

exception is a newly defined entity, diffuse midline glioma, H3 K27M-mutant. ${ }^{2)}$

Diffuse midline glioma, H3 K27M-mutant (Fig. 2)

This is an infiltrative, high-grade glioma with predominately astrocytic differentiation that occurs in a midline location, i.e., the thalamus, brainstem or spinal cord, harboring a $\mathrm{K} 27 \mathrm{M}$ mutation in either H3F3A or HIST1H3B/C. . $^{48,50)}$ This tumor predominately affects children but can also be seen in adults. It is classified as WHO grade IV regardless of the presence or absence of anaplastic features. ${ }^{2)}$

\section{Ependymomas}

There have been few changes in the nomenclature related to ependymomas in this revision, since the recently proposed molecular classification of ependymomas is based on DNA methylation profiling, which is only available in restricted institutions. ${ }^{58)}$ One genetically-defined ependymoma subtype, ependymoma, RELA fusion-positive, has been accepted. The genetic alteration of this subtype is detectable by fluorescence in situ hybridization (FISH). ${ }^{59,60)}$ This variant accounts for the majority of supratentorial examples. The expression of L1cell adhesion molecule (CAM) is well correlated with the presence of a RELA fusion in supratentorial ependymomas but this is also expressed by other tumors. ${ }^{59)}$

Neuronal and mixed neuronal-glial tumors (Fig. 3)

Two lesions, diffuse leptomeningial glioneuronal tumor (DLGNT) ${ }^{61-63)}$ and multinodular and vacuolating neuronal tumor (MVNT), ${ }^{64-66)}$ both of which are considered to be unique lesions, have been described by various similar 

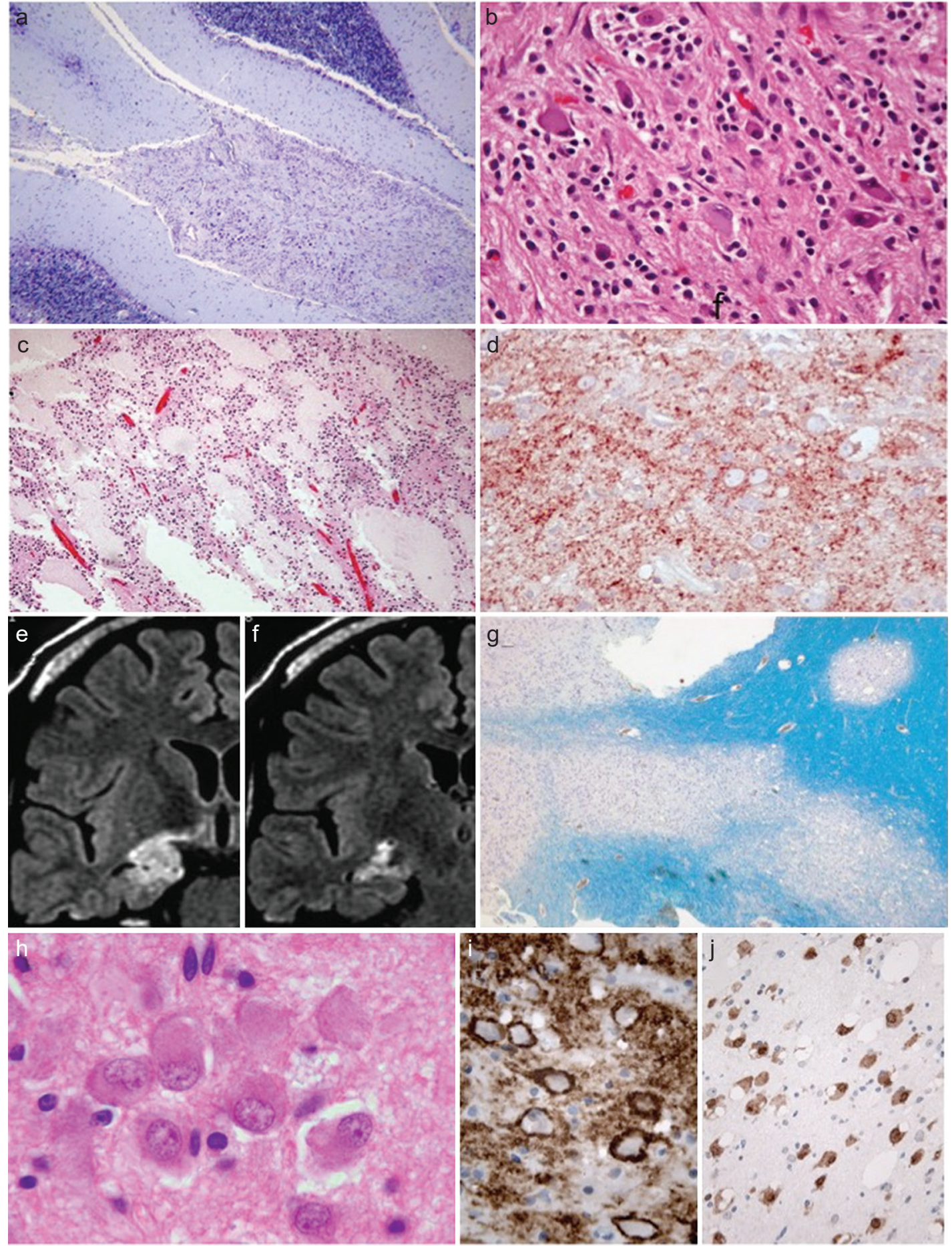

Fig. 3 Diffuse leptomeningial glioneuronal tumor (DLGNT) (a-d) and vacuolating neuronal tumor (MVNT) (e-j). (a) Expansion by tumor tissue of the cerebellar leptomeninges without apparent intraparenchymal masses (Klüver-Barrera staining). (b) Showing the mixture of small, round oligodendroglia-like cells and irregularly oriented neuronal cells. (c) Occasionally tumor tissue shows mucin-rich microcystic background. (d) Neuronal cells as well as the neoplastic stroma show positive synaptophysin immunoreactivity. (e, f) Axial FLAIR MRIs show a irregular cortical lesion in the right medial temporal lobe. (g) Multiple nodular or patchy lesions in the subcortical white matter are evident in Klüver-Barrera staining. (h) Dysplastic cells having an abundant amphiphilic to eosinophilic cytoplasm with peripheral Nissl substance showed focal clustering. (i). Tumor cells are strongly positive for $\alpha$-internexin on the cell membranes. (j) The dysplastic neurons were intensely stained by $\mathrm{HuC} / \mathrm{Hu}$. 
terms in the literature. DLGNT is characterized by the diffuse involvement of the leptomeninges, particularly those of the spinal cord, with or without recognizable parenchymal components. The major constituent of DLGNT is oligodendroglia-like cells with variable neuronal components (from neurocytes to ganglioid cells). DLGNT often poses BRAF fusions as well as chromosome $1 \mathrm{p}$ deletions. ${ }^{63)}$ MVNT is a quasi-tumor that is characterized by multiple nodules composed of vacuolating dysplastic neurons in the subcortical white matter. A relatively restrictive-either nodular or ribbon-like-growth pattern suggests that MVNT has a hamartomatous nature. ${ }^{66)}$

\section{Embryonal tumors}

The main changes in this category included the addition of medulloblastomas, which are genetically defined, and embryonal tumor with multilayered rosettes (ETMR), C19MC-altered. Central Nervous System (CNS)-primitive neuroectodermal tumor (PNET) was eliminated. For medulloblastoma, the most popular 4-type classification was not adopted in this revision; ${ }^{38,67)}$ however, WNT-activated, SHH-activated and non-WNT/SHH have been accepted instead. The SHH-activated tumors were divided into those with and without TP53 mutations that can be detected by immunohistochemistry. ${ }^{68)}$ Multilayered rosettes are characterized by a pseudostratified neuroepithelium with a central lumen covered by a defined apical surface with an internal limiting membrane; rosettes of this type always lack a defined outer membrane. ${ }^{69,70)}$ Multilayered rosettes are not always present in ETMR, C19MC-altered but medulloepithelioma-type rosettes may be present. Of note, a small portion of medulloepithelioma may harbor C19MC-alteration. ${ }^{71}$ If no diagnostic genetic alteration is identified, the tumor is classified as plain "medulloepithelioma".

DNA methylation profiling has revealed that majority of CNS-PNETs display molecular profiles indistinguishable from those of various other welldefined CNS tumor entities, which strongly suggests that CNS-PNETs are not an entity. ${ }^{72)}$ In the remaining fractions, in which well-defined entities were excluded, some unknown tumors, one of which resembles CNS neuroblastoma, have been reported, the details of those unknown tumors remain unclear. ${ }^{72)}$

\section{Immunohistochemical surrogates in a clinical setting}

Although WHO2016 does not allow the use of surrogate markers to detect molecular alterations, some hospitals/medical centers, particularly those located in areas other than Europe and North America, do not have full access to methods to detect the signature molecular alterations. ${ }^{73)}$ In the clinical setting, the use of immunohistochemical surrogates is necessary. ${ }^{74)}$ Since Sanger sequencing, the most standard method to detect point mutations on $I D H 1 / 2$, requires at least $20 \%$ of mutant alleles for identifying mutations, ${ }^{75}$ immunohistochemistry can be more sensitive than genetic tastings. Nonetheless, it is important to bear in mind that no surrogate markers can be used as a substitute for an official WHO diagnosis and we have to facilitate departmental and institutional molecular testing to improve the diagnosis of brain tumors. The immunohistochemical surrogates that fulfill the WHO2016 diagnoses are shown in Table 5.

\section{Acknowledgment}

The author is deeply grateful to Dr. Masaaki Nitta, Dr. Takashi Maruyama, Dr. Yoshihiro Muragaki and Dr. Takakazu Kawamata and the members of Faculty of Advanced Techno-Surgery, Institute of Advanced Biomedical Engineering \& Science, Graduate School of Medicine, and Department of Neurosurgery, Neurological Institute, Tokyo Women's Medical University for their generous supports and valuable discussions. The author is also grateful to Mr. Brian Quinn for carefully proofreading the manuscript.

\section{Conflicts of Interest Disclosure}

The author declares no conflicts of interest.

\section{References}

1) Louis DN: The next step in brain tumor classification: "Let us now praise famous men"... or molecules? Acta Neuropathol 124: 761-762, 2012

2) Louis DN, Ohgaki H, Wiestler OD, Cavenee WK: WHO classification of tumours of the central nervous system. Revise 4th edition. Lyon: IARC Press, 2016

3) Louis DN, Perry A, Reifenberger G, et al.: The 2016 World Health Organization classification of tumors of the central nervous system: a summary. Acta Neuropathol 131: 803-820, 2016

4) Perry A: WHO's arrived in 2016! An updated weather forecast for integrated brain tumor diagnosis. Brain Tumor Pathol 33: 157-160, 2016

5) Bailey P, Cushing H: A classification of tumors of the glioma group on a histogenetic basis with a correlated study of prognosis. Philadelphia: JB Lippincott, 1926

6) Louis DN, Ohgaki H, Wiestler OD, Cavenee WK: WHO Classification of Tumours of the Central Nervous System. 4th edition. Lyon: IARC Press, 2007

7) Teo JG, Gultekin SH, Bilsky M, Gutin P, Rosenblum MK: A distinctive glioneuronal tumor of the adult cerebrum with neuropil-like (including "rosetted") 
islands: report of 4 cases. Am J Surg Pathol 23: 502-510, 1999

8) Perry A, Burton SS, Fuller GN, et al.: Oligodendroglial neoplasms with ganglioglioma-like maturation: a diagnostic pitfall. Acta Neuropathol 120: 237-252, 2010

9) Rodriguez FJ, Scheithauer BW, Robbins PD, et al.: Ependymomas with neuronal differentiation: a morphologic and immunohistochemical spectrum. Acta Neuropathol 113: 313-324, 2007

10) Brat DJ, Hirose Y, Cohen KJ, Feuerstein BG, Burger PC: Astroblastoma: clinicopathologic features and chromosomal abnormalities defined by comparative genomic hybridization. Brain Pathol 10: 342-352, 2000

11) Pasquier B, Péoc'h M, Morrison AL, et al.: Chordoid glioma of the third ventricle: a report of two new cases, with further evidence supporting an ependymal differentiation, and review of the literature. Am J Surg Pathol 26: 1330-1342, 2002

12) Jouvet A, Fauchon F, Liberski P, et al.: Papillary tumor of the pineal region. Am J Surg Pathol 27: 505-512, 2003

13) Wang M, Tihan T, Rojiani AM, et al.: Monomorphous angiocentric glioma: a distinctive epileptogenic neoplasm with features of infiltrating astrocytoma and ependymoma. J Neuropathol Exp Neurol 64: 875-881, 2005

14) Cairncross JG, Ueki K, Zlatescu MC, et al.: Specific genetic predictors of chemotherapeutic response and survival in patients with anaplastic oligodendrogliomas. J Natl Cancer Inst 90: 1473-1479, 1998

15) Ohgaki H, Kleihues P: Population-based studies on incidence, survival rates, and genetic alterations in astrocytic and oligodendroglial gliomas. J Neuropathol Exp Neurol 64: 479-489, 2005

16) Hartmann C, Hentschel B, Wick W, et al.: Patients with IDH1 wild type anaplastic astrocytomas exhibit worse prognosis than IDH1-mutated glioblastomas, and IDH1 mutation status accounts for the unfavorable prognostic effect of higher age: implications for classification of gliomas. Acta Neuropathol 120: 707-718, 2010

17) Cancer Genome Atlas Research Network, Brat DJ, Verhaak RG, Aldape KD, et al.: Comprehensive, integrative genomic analysis of diffuse lower-grade gliomas. $N$ Engl J Med 372: 2481-2498, 2015

18) Eckel-Passow JE, Lachance DH, Molinaro AM, et al.: Glioma groups based on 1p/19q, IDH, and TERT promoter mutations in tumors. $N$ Engl J Med 372: 2499-2508, 2015

19) Mur P, Mollejo M, Hernández-Iglesias T, et al.: Molecular classification defines 4 prognostically distinct glioma groups irrespective of diagnosis and grade. J Neuropathol Exp Neurol 74: 241-249, 2015

20) Reuss DE, Mamatjan Y, Schrimpf D, et al.: IDH mutant diffuse and anaplastic astrocytomas have similar age at presentation and little difference in survival: a grading problem for WHO. Acta Neuropathol 129: 867-73, 2015
21) Olar A, Wani KM, Alfaro-Munoz KD, et al.: IDH mutation status and role of WHO grade and mitotic index in overall survival in grade II-III diffuse gliomas. Acta Neuropathol 129: 585-596, 2015

22) Kros JM, Gorlia T, Kouwenhoven MC, et al.: Panel review of anaplastic oligodendroglioma from European Organization For Research and Treatment of Cancer Trial 26951: assessment of consensus in diagnosis, influence of $1 \mathrm{p} / 19 \mathrm{q}$ loss, and correlations with outcome. J Neuropathol Exp Neurol 66: 545-551, 2007

23) Giannini C, Burger PC, Berkey BA, et al.: Anaplastic oligodendroglial tumors: refining the correlation among histopathology, 1p 19q deletion and clinical outcome in Intergroup Radiation Therapy Oncology Group Trial 9402. Brain Pathol 18: 360-369, 2008

24) van den Bent MJ: Interobserver variation of the histopathological diagnosis in clinical trials on glioma: a clinician's perspective. Acta Neuropathol 120: 297-304, 2010

25) Reifenberger J, Reifenberger G, Liu L, James CD, Wechsler W, Collins VP: Molecular genetic analysis of oligodendroglial tumors shows preferential allelic deletions on $19 \mathrm{q}$ and 1p. Am J Pathol 145: 1175-1190, 1994

26) Ueki K, Nishikawa R, Nakazato Y, et al.: Correlation of histology and molecular genetic analysis of $1 \mathrm{p}$, 19q, 10q, TP53, EGFR, CDK4, and CDKN2A in 91 astrocytic and oligodendroglial tumors. Clin Cancer Res 8: 196-201, 2002

27) Giannini C, Scheithauer BW, Weaver AL, et al.: Oligodendrogliomas: reproducibility and prognostic value of histologic diagnosis and grading. I Neuropathol Exp Neurol 60: 248-262, 2001

28) Miller CR, Dunham CP, Scheithauer BW, Perry A: Significance of necrosis in grading of oligodendroglial neoplasms: a clinicopathologic and genetic study of newly diagnosed high-grade gliomas. J Clin Oncol 24: 5419-5426, 2006

29) Komori T, Hirose T, Shibuya M, Suzuki H, Tanaka S, Sasaki A: Controversies over the diagnosis of oligodendroglioma: a report from the satellite workshop at the 4th international symposium of brain tumor pathology, Nagoya Congress Center, May 23, 2012. Brain Tumor Pathol 30: 253-261, 2013

30) Takahashi K, Tsuda M, Kanno H, et al.: Differential diagnosis of small cell glioblastoma and anaplastic oligodendroglioma: a case report of an elderly man. Brain Tumor Pathol 31: 118-123, 2014

31) Sahm F, Reuss D, Koelsche C, et al.: Farewell to oligoastrocytoma: in situ molecular genetics favor classification as either oligodendroglioma or astrocytoma. Acta Neuropathol 128: 551-559, 2014

32) Cairncross G, Wang M, Shaw E, et al.: Phase III trial of chemoradiotherapy for anaplastic oligodendroglioma: long-term results of RTOG 9402. J Clin Oncol 31: 337-343, 2013

33) van den Bent MJ, Brandes AA, Taphoorn MJ, et al.: Adjuvant procarbazine, lomustine, and vincristine chemotherapy in newly diagnosed anaplastic 
oligodendroglioma: long-term follow-up of EORTC brain tumor group study 26951. J Clin Oncol 31: 344-350, 2013

34) Yan H, Parsons DW, Jin G, et al.: IDH1 and IDH2 mutations in gliomas. $N$ Engl J Med 360: 765-773, 2009

35) Hartmann C, Meyer J, Balss J, et al.: Type and frequency of IDH1 and IDH2 mutations are Related to astrocytic and oligodendroglial differentiation and age: a study of 1,010 diffuse gliomas. Acta Neuropathol 2009

36) Balss J, Meyer J, Mueller W, Korshunov A, Hartmann C, von Deimling A: Analysis of the IDH1 codon 132 mutation in brain tumors. Acta Neuropathol 116: 597-602, 2008

37) Suzuki H, Aoki K, Chiba K, et al.: Mutational landscape and clonal architecture in grade II and III gliomas. Nat Genet 47: 458-468, 2015

38) Taylor MD, Northcott PA, Korshunov A, et al.: Molecular subgroups of medulloblastoma: the current consensus. Acta Neuropathol 123: 465-472, 2012

39) Kool M, Jones DT, Jäger N, et al.; ICGC PedBrain Tumor Project: Ge nome sequencing of SHH medulloblastoma predicts genotype-related response to smoothened inhibition. Cancer Cell 25: 393-405, 2014

40) Louis DN, Perry A, Burger P, et al.; International Society of Neuropathology-Haarlem: International Society of Neuropathology-Haarlem consensus guidelines for nervous system tumor classification and grading. Brain Pathol 24: 429-435, 2014

41) Reuss DE, Sahm F, Schrimpf D, et al.: ATRX and IDH1-R132H immunohistochemistry with subsequent copy number analysis and IDH sequencing as a basis for an "integrated" diagnostic approach for adult astrocytoma, oligodendroglioma and glioblastoma. Acta Neuropathol 129: 133-146, 2015

42) Capper D, Berghoff AS, Magerle M, et al.: Immunohistochemical testing of BRAF V600E status in 1,120 tumor tissue samples of patients with brain metastases. Acta Neuropathol 123: 223-233, 2012

43) Kato Y: Specific monoclonal antibodies against IDH1/2 mutations as diagnostic tools for gliomas. Brain Tumor Pathol 32: 3-11, 2015

44) Arita H, Narita Y, Yoshida A, Hashimoto N, Yoshimine T, Ichimura K: IDH1/2 mutation detection in gliomas. Brain Tumor Pathol 32: 79-89, 2015

45) Nguyen DN, Heaphy CM, de Wilde RF, et al.: Molecular and morphologic correlates of the alternative lengthening of telomeres phenotype in high-grade astrocytomas. Brain Pathol 23: 237-243, 2013

46) Abedalthagafi M, Phillips JJ, Kim GE, et al.: The alternative lengthening of telomere phenotype is significantly associated with loss of ATRX expression in high-grade pediatric and adult astrocytomas: a multi-institutional study of 214 astrocytomas. Mod Pathol 26: 1425-1432, 2013

47) Takami H, Yoshida A, Fukushima S, et al.: Revisiting TP53 mutations and immunohistochemistry-a comparative study in 157 diffuse gliomas. Brain Pathol 25: 256-265, 2015

48) Khuong-Quang DA, Buczkowicz P, Rakopoulos P, et al.: K27M mutation in histone H3.3 defines clinically and biologically distinct subgroups of pediatric diffuse intrinsic pontine gliomas. Acta Neuropathol 124: 439-447, 2012

49) Wu G, Broniscer A, McEachron TA, et al.; St. Jude Children's Research Hospital-Washington University Pediatric Cancer Genome Project: Somatic histone H3 alterations in pediatric diffuse intrinsic pontine gliomas and non-brainstem glioblastomas. Nat Genet 44: 251-253, 2012

50) Castel D, Grill J, Debily MA: Histone H3 genotyping refines clinico-radiological diagnostic and prognostic criteria in DIPG. Acta Neuropathol 131: 795-796, 2016

51) Zhang J, Wu G, Miller CP, et al.; St. Jude Children's Research Hospital-Washington University Pediatric Cancer Genome Project: Whole-genome sequencing identifies genetic alterations in pediatric low-grade gliomas. Nat Genet 45: 602-612, 2013

52) Wu G, Diaz AK, Paugh BS, et al.; St. Jude Children's Research Hospital-Washington University Pediatric Cancer Genome Project: The genomic landscape of diffuse intrinsic pontine glioma and pediatric non-brainstem high-grade glioma. Nat Genet 46: 444-450, 2014

53) Rodriguez FJ, Tihan T, Lin D, et al.: Clinicopathologic features of pediatric oligodendrogliomas: a series of 50 patients. Am J Surg Pathol 38: 1058-1070, 2014

54) Reuss DE, Kratz A, Sahm F, et al.: Adult IDH wild type astrocytomas biologically and clinically resolve into other tumor entities. Acta Neuropathol 130: 407-417, 2015

55) Ohgaki H, Kleihues P: The definition of primary and secondary glioblastoma. Clin Cancer Res 19: 764-772, 2013

56) Kleinschmidt-DeMasters BK, Alassiri AH, Birks DK, Newell KL, Moore W, Lillehei KO: Epithelioid versus rhabdoid glioblastomas are distinguished by monosomy 22 and immunohistochemical expression of INI-1 but not claudin 6. Am J Surg Pathol 34: 341-354, 2010

57) Kleinschmidt-DeMasters BK, Aisner DL, Birks DK, Foreman NK: Epithelioid GBMs show a high percentage of BRAF V600E mutation. Am J Surg Pathol 37: 685-698, 2013

58) Pajtler KW, Pfister SM, Kool M: Molecular dissection of ependymomas. Oncoscience 2: 827-828, 2015

59) Parker M, Mohankumar KM, Punchihewa C, et al.: C11orf95-RELA fusions drive oncogenic NF- $\mathrm{B}$ signalling in ependymoma. Nature 506: 451-455, 2014

60) Pietsch T, Wohlers I, Goschzik T, et al.: Supratentorial ependymomas of childhood carry C11orf95-RELA fusions leading to pathological activation of the NF-кB signaling pathway. Acta Neuropathol 127: 609-611, 2014 
61) Yamamoto T, Komori T, Shibata N, Toyoda C, Kobayashi M: Multifocal neurocytoma/gangliocytoma with extensive leptomeningeal dissemination in the brain and spinal cord. Am J Surg Pathol 20: 363-370, 1996

62) Rodriguez FJ, Perry A, Rosenblum MK, et al.: Disseminated oligodendroglial-like leptomeningeal tumor of childhood: a distinctive clinicopathologic entity. Acta Neuropathol 124: 627-641, 2012

63) Rodriguez FJ, Schniederjan MJ, Nicolaides T, Tihan T, Burger PC, Perry A: High rate of concurrent BRAF-KIAA1549 gene fusion and 1p deletion in disseminated oligodendroglioma-like leptomeningeal neoplasms (DOLN). Acta Neuropathol 129: 609-610, 2015

64) Huse JT, Edgar M, Halliday J, Mikolaenko I, Lavi E, Rosenblum MK: Multinodular and vacuolating neuronal tumors of the cerebrum: 10 cases of a distinctive seizure-associated lesion. Brain Pathol 23: 515-524, 2013

65) Nagaishi M, Yokoo H, Nobusawa S, et al.: Localized overexpression of alpha-internexin within nodules in multinodular and vacuolating neuronal tumors. Neuropathology 35: 561-568, 2015

66) Yamaguchi M, Komori T, Nakata Y, Yagishita A, Morino M, Isozaki E: Multinodular and vacuolating neuronal tumor affecting amygdala and hippocampus: A quasi-tumor? Pathol Int 66: 34-41, 2016

67) Ramaswamy V, Remke M, Bouffet E, et al.: Risk stratification of childhood medulloblastoma in the molecular era: the current consensus. Acta Neuropathol 131: 821-831, 2016

68) Zhukova N, Ramaswamy V, Remke M, et al.: Subgroup-specific prognostic implications of TP53 mutation in medulloblastoma. J Clin Oncol 31: 2927-2935, 2013
69) Judkins AR, Ellison DW: Ependymoblastoma: Dear, Damned, Distracting Diagnosis, Farewell!*. Brain Pathol 20: 133-139, 2008

70) Korshunov A, Remke M, Gessi M, et al.: Focal genomic amplification at 19q13.42 comprises a powerful diagnostic marker for embryonal tumors with ependymoblastic rosettes. Acta Neuropathol 120: 253-260, 2010

71) Spence T, Sin-Chan P, Picard D, et al.: CNS-PNETs with C19MC amplification and/or LIN28 expression comprise a distinct histogenetic diagnostic and therapeutic entity. Acta Neuropathol 128: 291-303, 2014

72) Sturm D, Orr BA, Toprak UH, et al.: New brain tumor entities emerge from molecular classification of CNS-PNETs. Cell 164: 1060-1072, 2016

73) Anderton JA, Lindsey JC, Lusher ME, et al.: Global analysis of the medulloblastoma epigenome identifies disease-subgroup-specific inactivation of COL1A2. Neuro-oncology 10: 981-994, 2008

74) Tanboon J, Williams EA, Louis DN: The Diagnostic Use of Immunohistochemical Surrogates for Signature Molecular Genetic Alterations in Gliomas. J Neuropathol Exp Neurol 75: 4-18, 2016

75) Arita H, Narita Y, Matsushita Y, et al.: Development of a robust and sensitive pyrosequencing assay for the detection of IDH1/2 mutations in gliomas. Brain Tumor Pathol 32: 22-30, 2015

Address reprint requests to: Takashi Komori, MD, PhD, Department of Laboratory Medicine and Pathology (Neuropathology), Tokyo Metropolitan Neurological Hospital, 2-6-1 Musashidai, Fuchu, Tokyo 183-0042, Japan.

e-mail: komori-tk@igakuken.or.jp 